\title{
Voice of NESON
}

$\mathrm{V}$

ery recently we have had a very successful, informative and enjoyable $1^{\text {st }}$ International and $5^{\text {th }}$ Annual Conference of Nepalese Society of Neurosurgeons. Eminent neurosurgeons, masters on their own fields, took their time off and came for this conference to be with us. We also had a wonderful preconference cadaveric workshop where the Nepalese neurosurgeons actively participated and learnt a lot. We had neurosurgeons from 65 countries from different parts of the world in this conference. Besides the academic part, the social events were also very enjoyable. There was ample of time to interact with the prominent neurosurgeons who were there till the conclusion of the conference. Lot of Nepalese neurosurgeons took advantage of this opportunity to interact and build up relations with them making platform for development of their skill and career further. We also had chance to renew our contacts and relation with these teachers of neurosurgery. We made new friends and opened a new era of opportunities.

From this conference, we made our bond stronger with international neurosurgical organizations like WFNS, ACNS, AANS, EANS, NSI and others. We got promises to get guidance, help and offer training to young neurosurgeons as well as assurance to continue to support our organization. We have already got calls for the young neurosurgeon to participate in several conferences including 13th ICCVS in Nagoya from Oct 27th to $29^{\text {th }}$ 2017 along with significant help to attend the conference. Now it is up to us how much advantage, individually and organization wise, we can extract from these relationship.

The regular activities of NESON haveagain resumed with continuity of the regular $\mathrm{CME}$ of NESON. In April, Pokhara under the leadership of Dr. BalGopalKarmacharya,is organizing the CME on the eve of Nepali New Year. In addition, on the same day he is also organizing the CME and a workshop on behalf of Spine Chapter of Nepalese Society of Neurosurgeons at Manipalteaching Hospital. The Editors of our Journal, Nepal Journal of Neuroscience, are working very hard, with lot of success; to timely bring out a better quality journal and we all applaud for their effort.

Dear friends we are together moving forward and lets continue doing that. Using social media network like viber, we are discussing and sharing cases, increasing our knowledge and bondage. The culture of seniors helping the juniors to promote them and develop their skill using one's resources is very evident in our society that promises a better future of the neurosurgeons of Nepal.

Executive Committee

Nepalese Society of Neurosurgeon 